\title{
Erratum: Pulmonary Embolism and Atrial Fibrillation: Two Sides of the Same Coin? A Systematic Review
}

\author{
Behnood Bikdeli, MD ${ }^{1,2,3}$ Maen D. Abou Ziki, MD ${ }^{4}$ Gregory Y. H. Lip, MD 5,6
}

${ }^{1}$ Division of Cardiology, Department of Medicine, Columbia University Medical Center, New York, New York

2 New York-Presbyterian Hospital, New York, New York

${ }^{3}$ Center for Outcomes Research and Evaluation, Yale University School of Medicine, New Haven, Connecticut

${ }^{4}$ Yale University School of Medicine, New Haven, Connecticut

${ }^{5}$ University of Birmingham Institute of Cardiovascular Sciences, City Hospital, Birmingham, United Kingdom

${ }^{6}$ Thrombosis Research Unit, Aalborg University, Aalborg, Denmark

Semin Thromb Hemost 2018;43:e1.

Address for correspondence Behnood Bikdeli, MD, Center for Outcomes Research and Evaluation, Yale University School of Medicine, 1 Church Street, Suite 200, New Haven, CT 06510 (e-mail: Behnood.bikdeli@yale.edu; bb2813@cumc.columbia.edu).

\section{ERRATUM}

It has been brought to our attention that the name of Maen D. Abou Ziki was indexed incorrectly in the above article published online ahead of print on February 14, 2017, in the journal Seminars in Thrombosis \& Hemostasis (DOI: 10.1055/s-0036-1598005). The author's name was incorrectly indexed as "Ziki Maen D" but the correct listing should appear as "Abou Ziki, Maen D" or as "Abou Ziki MD." Guest Editors: Sam Schulman, MD, PhD, and Md. Shahidul Islam, MD, PhD. 